\title{
Selection of an Appropriate 'Statistical Model' for Baseline Road for Low Volume Traffic Flow
}

\section{K. S. Weerasekera}

\begin{abstract}
It was attempted to select an appropriate 'statistical model' for a set of observed traffic data on Baseline road, Colombo. A traffic counting survey was conducted to collect traffic data along Baseline road on a weekend. A Sunday afternoon was selected to conduct the traffic survey to ensure an uninterrupted flow condition without congestion at the site. Data obtained from the survey was analysed and appropriate counting interval and time period for the development of the model were selected.
\end{abstract}

A counting interval of 10 seconds was conducted, and later a spectrum of $10,20 \& 30$ second intervals were computed and plotted. It was, observed that 10 second counting interval gave the best shape graph with a maximum frequency at one vehicle per 10 second interval and a positive skew. The observed frequency distribution for 10 second interval, showed a reasonably well distribution with a single mode, and a positive skew tailing to the right. The 20 and 30 second intervals showed a deviation from the regular pattern further and further. Hence the pattern seems to deteriorate with the increasing time interval.

The analysis proved that the sample could be identified as Negative Binomially distributed. A goodness-of-fit test was used to check the difference between field data and expected frequency. Negative Binomial Model (with $k=2$ ) gave the smallest Chi-square value with parameters of $p=0.38$, which best fitted to the obtained sample data. Hence it could be concluded that Baseline road traffic was 'randomly' distributed under uninterrupted flow conditions, and Negative Binomial Model was the statistical model that best described the traffic flow conditions at the site at the time.

Keywords: Traffic Flow Modelling, Negative Binomially Distribution

\section{Introduction}

The aim of this study is to select an appropriate 'statistical model' for a set of observed traffic data on Baseline road, Colombo. A traffic counting survey was conducted to collect traffic data along Baseline road on a weekend. Sunday the $18^{\text {th }}$ January 2009 afternoon was selected to conduct the traffic survey to ensure an uninterrupted flow condition without congestion at the site. Data obtained from the survey was analysed and appropriate counting interval and time period for the development of the model were selected.

\section{Data Collection}

The counting site (Figure 1) was selected on Baseline road at the Southern end of the flyover (across the main railway line). This section of the road is a divided road with uninterrupted traffic flow without any side friction from roadside activities. The traffic survey was carried out on a Sunday afternoon $(2: 00 \mathrm{pm}$ to $3: 00 \mathrm{pm})$ for duration of 60 minutes. The count was performed on north-bound middle lane traffic which seemed to be fairly steady.

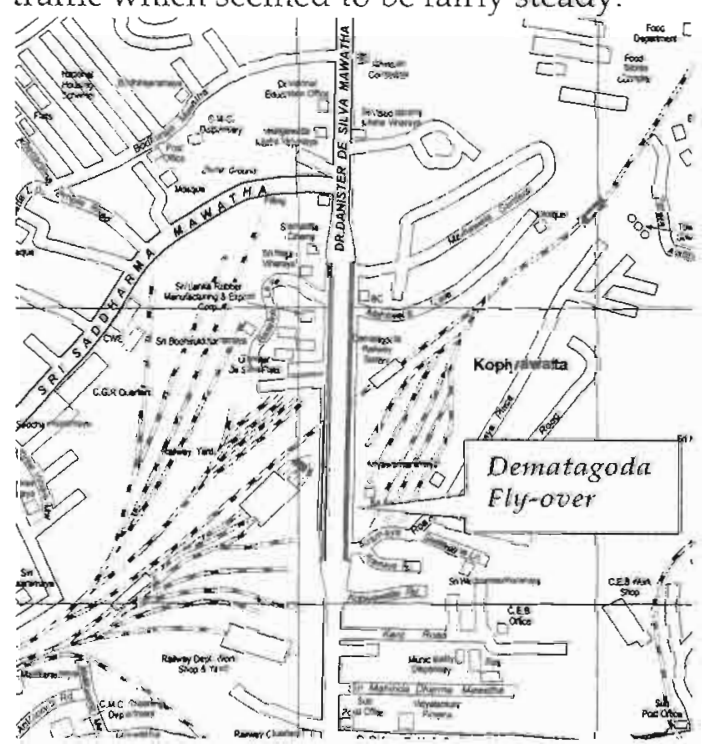

Figure 1 - Survey site
Eng. (Prof.) K. S. Weerasekera, BSc Eng (Moratuwa), MEngSc (UNSW), PhD (UNSW), FIE (Sri Lanka), CEng, MIE (Aust), CPEng, MIHT (LIK), MASCE, Professor in Civil Engineering, The Open University of Sri Lankn Lanka. 
Number of vehicles travelling in the middle lane towards north (i.e., from Borella to Urugodawatta direction) was recorded at 10 second intervals (Appendix 1). When deciding on the counting interval, it was ensured that the interval was such that it was humanly possible to record them at the field, and also able to manipulate the data to obtain a spectrum of intervals (10 seconds, 20 seconds, 30 seconds etc.).

\section{Data Analysis}

The frequency distributions were plotted for a spectrum of counting intervals of 10 seconds, 20 seconds, and 30 seconds as illustrated in Figures, 2, 3, and 4.

The observed frequency distribution for 10 second interval shows reasonably well a distribution with a single mode, and a positive skew tailing to the right (Figure 2). It can be seen from Figures 3 and 4, for 20 second interval and 30 second interval, shapes deviate from the regular pattern further and further. Hence the pattern seems to deteriorate with the increasing counting interval.

Out of the three frequency distribution graphs, the 10 second interval graph (Figure 2) which had the best shape with a positive skew and a maximum frequency, was selected for the development of a counting model.

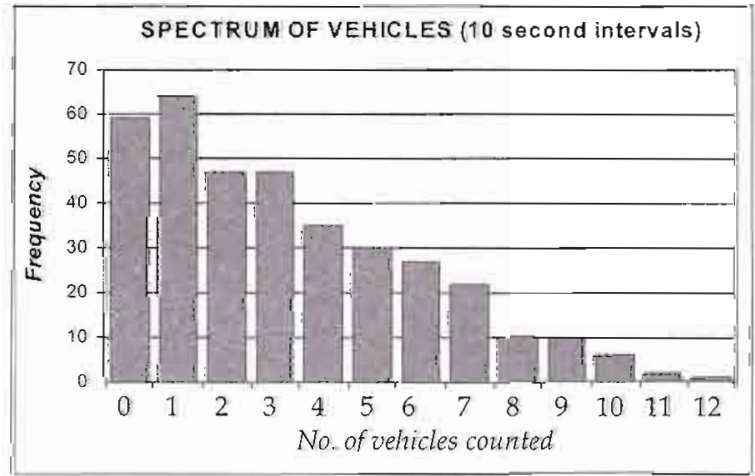

Figure 2 - Spectrum of vehicles at 10 second intervals

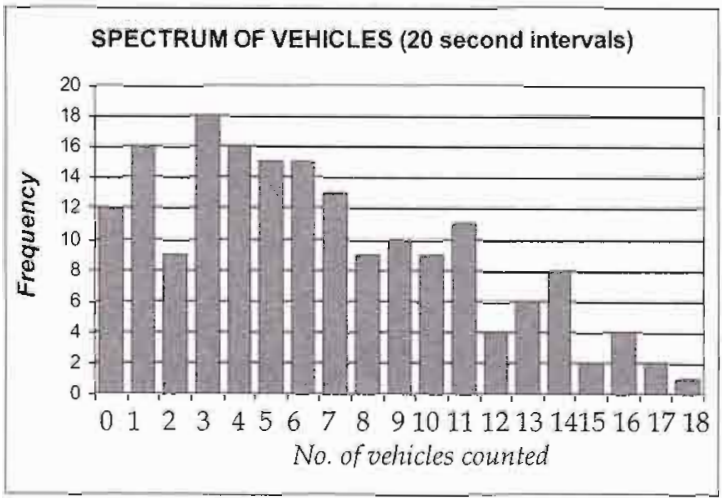

Figure 3 - Spectrum of vehicles at 20 second intervals

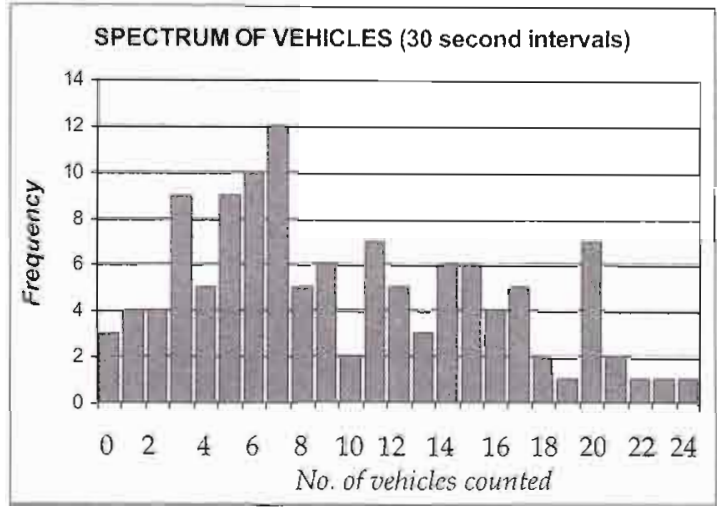

Figure 4 - Spectrum of vehicles at 30 second intervals

Statistical analysis for the selected time period $(2: 00 \mathrm{pm}$ to $3: 00 \mathrm{pm})$ at 10 second counting interval is indicated in Table 1.

Table 1 - Statistical Analysis

\begin{tabular}{|c|c|c|c|}
\hline Count $(\boldsymbol{x})$ & $\begin{array}{c}\text { Frequency } \\
\left(\mathbf{f}_{\mathbf{x}}\right)\end{array}$ & $\mathbf{x} \mathbf{\mathbf { f } _ { \mathbf { x } }}$ & $\mathbf{x}^{\mathbf{2}} \cdot \mathbf{f}_{\mathbf{x}}$ \\
\hline 0 & 59 & 0 & 0 \\
1 & 64 & 64 & 64 \\
2 & 47 & 94 & 188 \\
3 & 47 & 141 & 423 \\
4 & 35 & 140 & 560 \\
5 & 30 & 150 & 750 \\
6 & 27 & 162 & 972 \\
7 & 22 & 154 & 1078 \\
8 & 10 & 80 & 640 \\
9 & 10 & 90 & 810 \\
10 & 6 & 60 & 600 \\
11 & 2 & 22 & 242 \\
12 & 1 & 12 & 144 \\
$>12$ & 0 & 0 & 0 \\
\hline TOTAL & 360 & $\mathbf{1 1 6 9}$ & $\mathbf{6 4 7 1}$ \\
\hline
\end{tabular}




\subsection{Statistics:}

$$
\begin{aligned}
\text { Sample mean } & =\bar{x}=\frac{\sum x \cdot f_{x}}{\sum f_{x}} \\
& =1169 / 360=3.247 \\
\text { Mean of square } & =\overline{x^{2}}=\frac{\sum x^{2} \cdot f_{x}}{\sum f_{x}} \\
& =6471 / 360=17.975
\end{aligned}
$$

Sample variance $=s^{2}=\overline{x^{2}}-(\bar{x})^{2}$

$$
=17.975-(3.247)^{2},=7.432
$$

Standard deviation $=s=(7.432)^{1 / 2}=2.726$

Index of dispersion $=I=\frac{s^{2}}{\bar{x}}=7.432 / 3.247$

$$
=2.289
$$

Since, $\bar{x}=3.247$ veh $/ 10$ seconds;

traffic volume per hour $=3.247 \times 6 \times 60$

$$
=1169 \mathrm{veh} / \mathrm{hr} \text {. }
$$

\subsection{Choice of Model}

Index of dispersion (I) value was used for the selection of the model. If, $\mathrm{I}=1$ or approximately equal to 1, Poisson model may sometimes be suitable, but not always (Adams, [1]). If, $\mathrm{I}<1$, Binomial model may be suitable. This has been further justified by Mannering et al. [3].

As for the obtained data, $I=2.289$ which is greater than 1, Negative Binomial distribution is analysed for selection, as suggested by Gosling[2] and Walpole and Myers [4].

\section{Negative Binomial Distribution}

$$
\begin{gathered}
p(X=x)=\left(\frac{x+k-1}{k-1}\right) p^{k}(1-p)^{x} \\
x=0,1,2, \ldots \ldots \\
p_{x+1}=\left(\frac{x+k}{x+1}\right)(1-p) p_{x} \\
x \geq 0
\end{gathered}
$$

$$
\hat{k}=\frac{\bar{x}}{I-1}=\frac{3.247}{(2.289-1)}=2.5190
$$

Nearest integers are $\hat{k}=2$ and $\hat{k}=3$

Let's try, $\hat{k}=2$ and $\hat{k}=3$

(1) when, $k=2$

Hence,

$$
\begin{aligned}
& \hat{p}=\frac{k}{\bar{x}+k}=\frac{2}{(3.247+2)}=0.3812 \\
& p_{x+1}=\left(\frac{x+k}{x+1}\right)(1-p) p_{x} \quad x \geq 0 \\
& p_{0}=p^{k}=(0.3812)^{2}=0.1453 \\
& e_{0}=360 \times p_{0}=(360) \times 0.1453 \\
& e_{x} \text { - expected frequency } \\
& f_{x} \text { - observed frequency }
\end{aligned}
$$

Calculation and results of $e_{x}$ and $\chi_{x}^{2}$ is given in Table 2.

(1) when, $k=3$

Hence,

$$
\begin{aligned}
& \hat{p}=\frac{k}{\bar{x}+k}=\frac{3}{(3.247+3)}=0.4802 \\
& p_{x+1}=\left(\frac{x+k}{x+1}\right)(1-p) p_{x} \quad \mathrm{x} \geq 0 \\
& p_{0}=p^{k}=(0.4802)^{3}=0.1107 \\
& e_{0}=360 \times p_{0}=(360) \times 0.1107 \\
& e_{x} \text { - expected frequency } \\
& f_{x} \text { - observed frequency }
\end{aligned}
$$

Calculation and results of $e_{x}$ and $\chi_{x}^{2}$ is given in Table 2.

Estimated the following parameters,

$\hat{p}=\mathrm{I}^{-1}=1 / 2.289=0.4369$ 


\section{Results}

Table 2 - Chi-square Test Analysis for $\mathrm{k}=2$ and $k=3$

\begin{tabular}{|c|c|c|c|c|c|}
\hline \multirow{2}{*}{$\begin{array}{c}\text { Count } \\
(\mathrm{x})\end{array}$} & \multirow{2}{*}{$\begin{array}{c}\text { Freq. } \\
\left(\mathrm{f}_{\mathrm{x}}\right)\end{array}$} & \multicolumn{2}{|c|}{$\mathrm{k}=2$} & \multicolumn{2}{c|}{$\mathrm{k}=3$} \\
\hline 0 & 59 & 52.31 & 0.856 & 39.86 & 9.191 \\
\hline 1 & 64 & 64.74 & 0.008 & 62.15 & 0.055 \\
\hline 2 & 47 & 60.08 & 2.848 & 64.60 & 4.795 \\
\hline 3 & 47 & 49.57 & 0.133 & 55.95 & 1.432 \\
\hline 4 & 35 & 38.34 & 0.291 & 43.62 & 1.703 \\
\hline 5 & 30 & 28.47 & 0.082 & 31.75 & 0.096 \\
\hline 6 & 27 & 20.56 & 2.017 & 22.01 & 1.131 \\
\hline 7 & 22 & 14.54 & 3.827 & 14.70 & 3.625 \\
\hline 8 & 10 & 10.12 & 0.001 & 9.54 & 0.022 \\
\hline 9 & 10 & 6.96 & 1.328 & 6.06 & 2.562 \\
\hline 10 & 6 & 4.73 & 0.341 & 3.77 & 1.319 \\
\hline 11 & 2 & 3.18 & 0.438 & 2.32 & 0.045 \\
\hline 12 & 1 & 2.17 & 0.631 & 1.41 & 0.118 \\
\hline$>12$ & 0 & 4.23 & 4.230 & 2.26 & 2.265 \\
\hline$\Sigma$ & 360 & & 17.032 & & 28.360 \\
\hline
\end{tabular}

From Table 2 results it is evident that when $\mathrm{k}=2, \sum\left(e_{x}-f_{x}\right)^{2} / e_{x}=17.032$

and,

when $\mathrm{k}=3, \quad \sum\left(e_{x}-f_{x}\right)^{2} / e_{x}=28.360$

Since when $\mathrm{k}=2 \quad \sum\left(e_{x}-f_{x}\right)^{2} / e_{x}$ has a lower value than when $\mathrm{k}=3$.

Since Negative Binomial Model (with $\mathrm{k}=2$ ) gives the smaller value of $\sum \chi_{x}^{2}=17.032$

Therefore the curve when $k=2$ and $p=0.3812$ is a better fit among the two curves.

\section{Conclusion}

From the results it can be clearly seen that road traffic is 'randomly' distributed under uninterrupted conditions. The particular counting time interval which demonstrates this randomness is 10 second intervals, and the vehicular flow is around $1170 \mathrm{veh} / \mathrm{hr}$.
Therefore the chosen Negative Binomial Model is as follows:

$$
\begin{aligned}
& \begin{array}{l}
p(X=x)=\left(\frac{x+k-1}{k-1}\right) p^{k}(1-p)^{x} \\
x=0,1,2, \ldots \ldots . .
\end{array} \\
& p(X=x)=\left(\frac{x+1}{1}\right)(0.3812)^{2}(1-0.3812)^{\prime \prime} \\
& p(X=x)=0.1453(x+1) \times 0.6188^{x}
\end{aligned}
$$

It was seen that the frequency distribution for counting interval of 10 seconds represented a much improved shape of a graph and the 20 second and 30 second intervals deviated further and further from the regular pattern. It also gave the impression that 30 second interval sketch as developing towards a bi-modal shape. Therefore the regular pattern seemed to deviate from the expected shape, with increasing counting interval.

\section{References}

1. Adams, W. F., 'Road Traffic Considered as a Random Series', Journal of the Institution of Civil Engineers, Vol. 4, November, 1936, pp. $121-130$.

2. Gosling, J. 'Introductory Statistics', Pascal Press, Glebe, NSW, Australia, 1995.

3. Mannering, F. L. , Kilareski, W. P. and Washburn, S. S. Principles of Highway Engineering and Traffic Analysis, 3rd ed., John Wiley \& Sons, Inc. USA, 2005, pp. 146-147.

4. Walpole, R.E. and Myers, R.H. 'Probability and Statistics for Engineers and Scientists', Maxwell Macmillan International Editions, 1990. 
APPENDIX A - Field Data Sheet

\begin{tabular}{|c|c|c|c|c|c|c|c|c|c|c|c|}
\hline \multirow{2}{*}{$\begin{array}{r}\text { Time } \\
2: 00 \text { pm }\end{array}$} & \multirow{2}{*}{$\begin{array}{r}\frac{10 s}{10} \\
10\end{array}$} & \multirow{2}{*}{$\begin{array}{l}\text { No. of } \\
\text { veh. } \\
3\end{array}$} & Time & \multirow{2}{*}{\multicolumn{2}{|c|}{$\begin{array}{cc}10 \mathrm{~s} & \text { No. of } \\
\text { interval } & \text { veh. }\end{array}$}} & \multicolumn{3}{|c|}{$\begin{array}{ccc}10 \mathrm{~s} & \text { No. of } \\
\text { Time interval } & \text { vel. } \\
\end{array}$} & \multirow{2}{*}{$\frac{\text { Time }}{2: 27 \mathrm{pm}}$} & \multirow{2}{*}{$\begin{array}{r}10 \mathrm{~s} \\
\text { interval } \\
10\end{array}$} & \multirow{2}{*}{$\begin{array}{l}\begin{array}{c}\text { No. of } \\
\text { veh. }\end{array} \\
\frac{7}{7}\end{array}$} \\
\hline & & & 2:09pm & & & $2: 18 \mathrm{pm}$ & 10 & 3 & & & \\
\hline & 20 & 4 & & 20 & 1 & & 20 & 2 & & 20 & 2 \\
\hline & 30 & 6 & & 30 & 2 & & 30 & 3 & & 30 & 3 \\
\hline & 40 & 3 & & 40 & 2 & & 40 & 0 & & 40 & 3 \\
\hline & 50 & 0 & & 50 & 2 & & 50 & 2 & & 50 & 2 \\
\hline & 60 & 0 & & 60 & 3 & & 60 & 3 & & 60 & 1 \\
\hline 2:0lpn & 10 & 2 & 2:10pm & 10 & 7 & $2: 19 \mathrm{pm}$ & 10 & 5 & $2: 28 \mathrm{pm}$ & 10 & 3 \\
\hline & 20 & 7 & & 20 & 2 & & 20 & 8 & & 20 & 1 \\
\hline & 30 & 9 & & 30 & 6 & & 30 & 7 & & 30 & 3 \\
\hline & 40 & 2 & & 40 & 8 & & 40 & 7 & & 40 & 4 \\
\hline & 50 & 4 & & 50 & 5 & & 50 & 5 & & 50 & 2 \\
\hline & 60 & 3 & & 60 & 3 & & 60 & 1 & & 60 & 11 \\
\hline $2: 02 \mathrm{pm}$ & 10 & 5 & $2: 1 \mathrm{lpm}$ & 10 & 4 & $2: 20 \mathrm{pm}$ & 10 & 0 & $2: 29 \mathrm{pm}$ & 10 & 8 \\
\hline & 20 & 6 & & 20 & 1 & & 20 & 1 & & 20 & 5 \\
\hline & 30 & 6 & & 30 & 1 & & 30 & 2 & & 30 & 4 \\
\hline & 40 & 4 & & 40 & 2 & & 40 & 1 & & 40 & 2 \\
\hline & 50 & 4 & & 50 & 0 & & 50 & 4 & & 50 & 0 \\
\hline & 60 & 7 & & 60 & 2 & & 60 & 3 & & 60 & 1 \\
\hline $2: 03 \mathrm{pm}$ & 10 & 2 & $2: 12 \mathrm{pm}$ & 10 & 3 & $2: 21 \mathrm{pm}$ & 10 & 4 & $2: 30 \mathrm{pm}$ & 10 & 3 \\
\hline & 20 & 1 & & 20 & 2 & & 20 & 3 & & 20 & 3 \\
\hline & 30 & 2 & & 30 & 2 & & 30 & 4 & & 30 & 0 \\
\hline & 40 & 6 & & 40 & 6 & & 40 & 0 & & 40 & 1 \\
\hline & 50 & 4 & & 50 & 5 & & 50 & 0 & & 50 & 4 \\
\hline & 60 & 5 & & 60 & 1 & & 60 & 0 & & 60 & 6 \\
\hline $2: 04 \mathrm{pm}$ & 10 & 2 & $2: 13 \mathrm{pm}$ & 10 & 10 & $2: 22 \mathrm{pm}$ & 10 & 0 & $2: 31 \mathrm{pm}$ & 10 & 7 \\
\hline & 20 & 1 & & 20 & 10 & & 20 & 1 & & 20 & 9 \\
\hline & 30 & 3 & & 30 & 0 & & 30 & 0 & & 30 & 4 \\
\hline & 40 & 5 & & 40 & 2 & & 40 & 0 & & 40 & 1 \\
\hline & 50 & 8 & & 50 & 3 & & 50 & 5 & & 50 & 1 \\
\hline & 60 & 7 & & 60 & 1 & & 60 & 11 & & 60 & 1 \\
\hline $2: 05 \mathrm{pm}$ & 10 & 1 & $2: 14 \mathrm{pm}$ & 10 & 0 & $2: 23 \mathrm{pm}$ & 10 & 5 & $2: 32 \mathrm{pm}$ & 10 & 0 \\
\hline & 20 & 1 & & 20 & 1 & & 20 & 5 & & 20 & 1 \\
\hline & 30 & 1 & & 30 & 1 & & 30 & 4 & & 30 & 1 \\
\hline & 40 & 4 & & 40 & 4 & & 40 & 1 & & 40 & 1 \\
\hline & 50 & 4 & & 50 & 0 & & 50 & 1 & & 50 & 7 \\
\hline & 60 & 3 & & 60 & 9 & & 60 & 1 & & 60 & 4 \\
\hline $2: 06 \mathrm{pm}$ & 10 & 3 & $2: 15 \mathrm{pm}$ & 10 & 7 & $2: 24 \mathrm{pm}$ & 10 & 3 & $2: 33 \mathrm{pm}$ & 10 & 6 \\
\hline & 20 & 0 & & 20 & 4 & & 20 & 3 & & 20 & 2 \\
\hline & 30 & 2 & & 30 & 4 & & 30 & 3 & & 30 & 9 \\
\hline & 40 & 3 & & 40 & 0 & & 40 & 2 & & 40 & 6 \\
\hline & 50 & 3 & & 50 & 1 & & 50 & 5 & & 50 & 0 \\
\hline & 60 & 5 & & 60 & 0 & & 60 & 2 & & 60 & 0 \\
\hline $2: 07 \mathrm{pm}$ & 10 & 4 & $2: 16 \mathrm{pm}$ & 10 & 2 & $2: 25 \mathrm{pm}$ & 10 & 4 & $2: 34 \mathrm{pm}$ & 10 & 0 \\
\hline & 20 & 3 & & 20 & 2 & & 20 & 10 & & 20 & 2 \\
\hline & 30 & 0 & & 30 & 3 & & 30 & 3 & & 30 & 2 \\
\hline & 40 & 5 & & 40 & 0 & & 40 & 1 & & 40 & 1 \\
\hline & 50 & 3 & & 50 & 9 & & 50 & 1 & & 50 & 5 \\
\hline & 60 & 8 & & 60 & 5 & & 60 & 3 & & 60 & 3 \\
\hline $2: 08 \mathrm{pm}$ & 10 & 2 & $2: 17 \mathrm{pm}$ & 10 & 4 & $2: 26 \mathrm{pm}$ & 10 & 3 & $2: 35 \mathrm{pm}$ & 10 & 0 \\
\hline & 20 & 1 & & 20 & 0 & & 20 & 1 & & 20 & I \\
\hline & 30 & 0 & & 30 & 1 & & 30 & 10 & & 30 & 8 \\
\hline & 40 & 3 & & 40 & 2 & & 40 & 4 & & 40 & 6 \\
\hline & 50 & 2 & & 50 & 0 & & 50 & 7 & & 50 & 1 \\
\hline & 60 & 7 & & 60 & 0 & & 60 & 9 & & 60 & 0 \\
\hline
\end{tabular}


APPENDIX A - Field Data Sheet (Contd.)

\begin{tabular}{|c|c|c|c|c|c|c|c|c|}
\hline Time & $\begin{array}{c}10 \mathrm{~s} \\
\text { interval }\end{array}$ & $\begin{array}{c}\text { No. of } \\
\text { veh. }\end{array}$ & Time & $\begin{array}{c}10 \mathrm{~s} \\
\text { interval }\end{array}$ & $\begin{array}{c}\text { No. of } \\
\text { veh. }\end{array}$ & Time & $\begin{array}{c}10 \mathrm{~s} \\
\text { interval }\end{array}$ & $\begin{array}{c}\text { No. of } \\
\text { veh. }\end{array}$ \\
\hline \multirow[t]{6}{*}{$2: 36 \mathrm{pm}$} & 10 & 1 & \multirow[t]{6}{*}{$2: 45 \mathrm{pm}$} & 10 & 4 & \multirow[t]{6}{*}{$2: 54 \mathrm{pm}$} & 10 & 0 \\
\hline & 20 & 0 & & 20 & 7 & & 20 & 4 \\
\hline & 30 & 0 & & 30 & 3 & & 30 & 5 \\
\hline & 40 & 0 & & 40 & 5 & & 40 & 7 \\
\hline & 50 & 2 & & 50 & 5 & & 50 & 7 \\
\hline & 60 & 5 & & 60 & 6 & & 60 & 6 \\
\hline \multirow[t]{6}{*}{$2: 37 \mathrm{pm}$} & 10 & 12 & \multirow[t]{6}{*}{$2: 46 \mathrm{pm}$} & 10 & 6 & \multirow[t]{6}{*}{$2: 55 \mathrm{pm}$} & 10 & 9 \\
\hline & 20 & 6 & & 20 & 7 & & 20 & 7 \\
\hline & 30 & 7 & & 30 & 9 & & 30 & 4 \\
\hline & 40 & 3 & & 40 & 3 & & 40 & 0 \\
\hline & 50 & 5 & & 50 & 0 & & 50 & 1 \\
\hline & 60 & 3 & & 60 & 1 & & 60 & 6 \\
\hline \multirow[t]{6}{*}{$2: 38 \mathrm{pm}$} & 10 & 1 & \multirow[t]{6}{*}{ 2:47pm } & 10 & 1 & \multirow[t]{6}{*}{$2: 56 \mathrm{pm}$} & 10 & 6 \\
\hline & 20 & 1 & & 20 & 0 & & 20 & 0 \\
\hline & 30 & 2 & & 30 & 4 & & 30 & 2 \\
\hline & 40 & 4 & & 40 & 5 & & 40 & 1 \\
\hline & 50 & 2 & & 50 & 1 & & 50 & 2 \\
\hline & 60 & 2 & & 60 & 5 & & 60 & 7 \\
\hline \multirow[t]{6}{*}{$2: 39 \mathrm{pm}$} & 10 & 6 & \multirow[t]{6}{*}{$2: 48 \mathrm{pm}$} & 10 & 2 & \multirow[t]{6}{*}{$2: 57 \mathrm{pm}$} & 10 & 6 \\
\hline & 20 & 6 & & 20 & 5 & & 20 & 8 \\
\hline & 30 & 6 & & 30 & 8 & & 30 & 7 \\
\hline & 40 & 4 & & 40 & 4 & & 40 & 3 \\
\hline & 50 & 5 & & 50 & 1 & & 50 & 0 \\
\hline & 60 & 5 & & 60 & 2 & & 60 & 0 \\
\hline \multirow[t]{6}{*}{$2: 40 \mathrm{pm}$} & 10 & 1 & \multirow[t]{6}{*}{$2: 49 \mathrm{pm}$} & 10 & 1 & \multirow[t]{6}{*}{$2: 58 \mathrm{pm}$} & 10 & 3 \\
\hline & 20 & 1 & & 20 & 3 & & 20 & 6 \\
\hline & 30 & 0 & & 30 & 2 & & 30 & 1 \\
\hline & 40 & 0 & & 40 & 1 & & 40 & 0 \\
\hline & 50 & 1 & & 50 & 4 & & 50 & 1 \\
\hline & 60 & 2 & & 60 & 0 & & 60 & 4 \\
\hline \multirow[t]{6}{*}{$2: 41 \mathrm{pm}$} & 10 & 0 & \multirow[t]{6}{*}{$2: 50 \mathrm{pm}$} & 10 & 1 & $2: 59 \mathrm{pm}$ & 10 & 3 \\
\hline & 20 & 0 & & 20 & 2 & & 20 & 1 \\
\hline & 30 & 0 & & 30 & 2 & & 30 & 0 \\
\hline & 40 & 7 & & 40 & 8 & & 40 & 1 \\
\hline & 50 & 6 & & 50 & 6 & & 50 & 3 \\
\hline & 60 & 8 & & 60 & 5 & & 60 & 3 \\
\hline $2: 42 \mathrm{pm}$ & 10 & 10 & $2: 51 \mathrm{pm}$ & 10 & 5 & $3: 00 \mathrm{pm}$ & & \\
\hline & 20 & 3 & & 20 & 1 & & & \\
\hline & 30 & 1 & & 30 & 0 & & & \\
\hline & 40 & 0 & & 40 & 0 & & & \\
\hline & 50 & 0 & & 50 & 0 & & & \\
\hline & 60 & 1 & & 60 & 0 & & & \\
\hline 2:43pm & 10 & 3 & 2:52pm & 10 & 10 & & & \\
\hline & 20 & 7 & & 20 & 1 & & & \\
\hline & 30 & 5 & & 30 & 1 & & & \\
\hline & 40 & 6 & & 40 & 6 & & & \\
\hline & 50 & 2 & & 50 & 9 & & & \\
\hline & 60 & 3 & & 60 & 9 & & & \\
\hline 2:44pm & 10 & 6 & 2:53pm & 10 & 6 & & & \\
\hline & 20 & 0 & & 20 & 0 & & & \\
\hline & 30 & 0 & & 30 & 1 & & & \\
\hline & 40 & 0 & & 40 & 2 & & & \\
\hline & 50 & 4 & & 50 & 1 & & & \\
\hline & 60 & 2 & & 60 & 4 & & & \\
\hline
\end{tabular}

